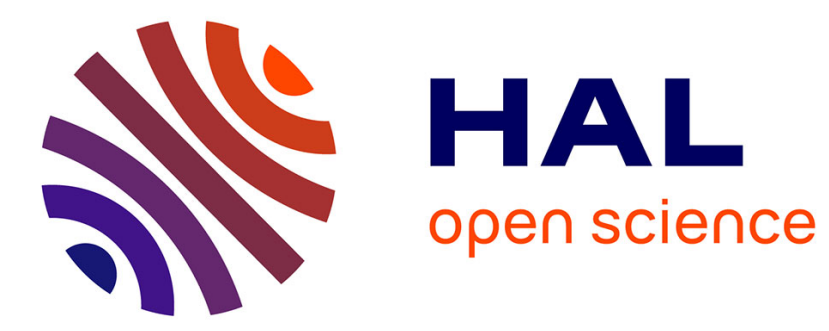

\title{
Les victimes entre émotions et stratégies
}

Christophe Traïni

\section{To cite this version:}

Christophe Traïni. Les victimes entre émotions et stratégies. Sandrine Lefranc et Lilian Mathieu (dir.), Mobilisations de victimes, 2009. halshs-02864027

\section{HAL Id: halshs-02864027 https://shs.hal.science/halshs-02864027}

Submitted on 10 Jun 2020

HAL is a multi-disciplinary open access archive for the deposit and dissemination of scientific research documents, whether they are published or not. The documents may come from teaching and research institutions in France or abroad, or from public or private research centers.
L'archive ouverte pluridisciplinaire HAL, est destinée au dépôt et à la diffusion de documents scientifiques de niveau recherche, publiés ou non, émanant des établissements d'enseignement et de recherche français ou étrangers, des laboratoires publics ou privés. 
Christophe TRAÏNI, « Les victimes entre émotions et stratégies », dans Sandrine Lefranc et Lilian Mathieu (dir.), Mobilisations de victimes, Presses Universitaires de Rennes, 2009.

\section{Christophe Traïni}

\section{Les victimes entre émotions et stratégies}

«VICTIME. s. f. On appelloit ainsi chez les Payens les animaux destinez pour estre offerts en sacrifice à leurs Dieux (...). On appelle, Nostre Seigneur Jesus-Christ la victime offerte pour le salut des hommes. Et dans le Sacrifice de la Messe, L'Hostie est appellée la Victime non sanglante (...). On appelle fig. Victimes, Les personnes qui sont consacrées à Dieu par des voeux solemnels. Cette fille a esté une victime agreable à Dieu ${ }^{1}$. L'étymologie du terme «victime» indique indubitablement que la notion ne peut être à l'origine dissociée de pratiques liturgiques fortement valorisées visant à manifester l'amour, la gratitude, la confiance absolue, la dévotion, à l'égard de divinités suscitant le plus grand respect. Ce n'est que très progressivement que l'expression a fini par désigner une personne qui souffre injustement du fait de la méchanceté, de l'injustice et de la haine illégitime d'un bourreau ignoble et méprisable. Alors même qu'il s'agissait autrefois d'évoquer un amour inconditionnel des plus louables, «se poser en victime » est devenu cet ensemble de conduites consistant à affirmer être traité avec injustice, à accuser un tortionnaire, à susciter l'indignation, à exiger enfin une réparation. Cette simple donnée étymologique attire notre attention sur le fait que les conduites des acteurs se réclamant de la catégorie de « victime » méritent sans doute d'être rapportées à la lente évolution historique des sensibilités collectives qui président à la formalisation des émotions au sein de l'espace public. Autant dire qu'une perspective inspirée des travaux de Norbert Élias parait, une fois de plus, fort utile afin de mieux saisir ce que la sociogenèse et la diffusion croissante des registres victimaires doivent à l'interdépendance d'un ensemble d'évolutions que l'on tendrait trop souvent à artificiellement dissocier. D'une part, la tendance séculaire au monopole de la violence au sein de ces institutions étatiques qui favorisent le règlement des conflits selon des voies politiques et plus encore judiciaires ${ }^{2}$. D'autre part, l'abaissement du seuil de sensibilité, non seulement à l'égard de l'exposition de soi à des agressions imprévisibles, mais encore à l'égard du spectacle de la violence qui porte atteinte au corps et à la dignité autrui. C'est d'ailleurs ce dernier élément qui favorisa le développement à partir du milieu XVIII ${ }^{\mathrm{e}}$ siècle de ce que Luc Boltanski — à la suite d'Annah Arendt — décrit comme une "politique de la pitié $»^{3}$. Dans la mesure où il pointe en direction d'une histoire nonoccidentale à l'origine d'un «art du massacre » sikh, le texte de Laurent Gayer qui va suivre nous rappelle la nécessité de cette contextualisation sociohistorique qui nous autorise à envisager les mobilisations de victimes comme l'une des étapes les plus récentes de cette euphémisation tendancielle des conduites agonistiques d'où résulta préalablement l'institutionnalisation, non seulement des arènes parlementaires et judiciaires, mais encore du combat électoral et de l'affaire menée en justice 4 .

Certes, il ne pourrait s'agir ici de postuler une inéluctable et implacable loi de l'histoire. Bien au contraire, les descriptions empiriques très fines qui étayent les chapitres à venir démontrent à quel point l'issue des interactions complexes sur lesquelles pèsent les sensibilités collectives héritées d'une histoire commune demeure irrémédiablement imprévisible. Bien plus encore, l'analyse quasi-ethnographique déployée par les auteurs permet d'entrevoir à quel point les mobilisations de victimes impliquent très concrètement — et conformément à l'étymologie du

\footnotetext{
${ }^{1}$ Dictionnaire de L'Académie française, première édition, 1694, p. 638.

${ }^{2}$ Les formes judiciaires du procès constituent le mode le plus courant d'octroi du statut de victime tant et si bien que la catégorie paraît désormais entretenir de fortes relations d'affinités avec le registre juridique valorisé au sein des «États de droit».

${ }^{3}$ Boltanski Luc, La souffrance à distance. Morale humanitaire, médias et politiques, Paris, Métailié, 1993, p. 15.

4 Voir, entre autres, Brigitte Gaïti et Liora Israël, "Sur l'engagement du droit dans la construction des causes », Politix, $\mathrm{n}^{\circ}$ 62, 2003. Sur les opérations très concrètes qui permettent aux acteurs des institutions judiciaires de transmuer les conflits entre requérants en des problématiques d'ordre juridique, voir également Bruno Latour, La fabrique du droit. Une ethnographie du Conseil d'État, Paris, La Découverte, 2002
} 
Christophe TRAÏNI, « Les victimes entre émotions et stratégies », dans Sandrine Lefranc et Lilian Mathieu (dir.), Mobilisations de victimes, Presses Universitaires de Rennes, 2009.

terme - des «liturgies », ou si l'on préfère des dispositifs de sensibilisation, qui invitent ceux qui les observent à des formes d'émotions dont la diversité se doit d'être soigneusement relevée 5 . De la dévotion, pourquoi pas ? Mais aussi de l'indignation, de la colère, du remord, de la culpabilité, de la compassion, du recueillement, de la nostalgie, de la fierté, de la joie, etc... Les commémorations de l'explosion de l'usine AZF, les évocations de l'assaut du Temple d'or ne se limitent jamais à en appeler à une catégorie de victime afin de faire valoir — d'un point de vue exclusivement cognitif — une certaine définition d'un évènement passé d'où résulte une forme d'attribution de rôles respectifs («Voilà ce qui s'est passé! Voilà qui furent les victimes, voilà qui furent les bourreaux!»). Bien plus fondamentalement, les conduites mises en œuvre au cours des mobilisations étudiées se présentent sous les traits d'entreprises qui visent à prescrire la nature et le degré d'émotions sans lesquelles les formulations argumentatives avancées s'avèreraient incapables de toucher les publics interpellés. Dans les cas traités par les auteurs, les entrepreneurs des causes - qui peuvent agir aussi bien « en tant que victimes » qu'au « nom des victimes »s'emploient avant tout à susciter auprès de leurs contemporains un certain nombre d'émotions susceptibles de les aider à faire valoir les revendications qu'ils adressent aux systèmes de la décision politique.

Affirmer ainsi que les acteurs étudiés s'emploient à prescrire des émotions susceptibles d'être partagées par le plus grand nombre revient, bien évidemment, à se demander aussitôt quel statut accorder à ces entreprises qui nécessitent la mobilisation de moyens, l'élaboration de stratégies appropriées, et qui peuvent très inégalement aboutir. Ici deux figures interprétatives pourraient rapidement s'imposer dès lors qu'il s'agit de rendre compte des rapports entre l'expression publique des émotions et les stratégies déployées afin de peser sur des décisions politiques : d'une part le dévoilement de conduites affectées, d'autre part le voile de la pudeur. Un premier type d'interprétation, en effet, consisterait à envisager les émotions manifestées et prescrites comme des moyens au service d'une fin stratégique prééminente: attirer l'attention des médias, recueillir des fonds auprès des publics émus par les dispositifs mis en place, légitimer des causes incertaines, et surtout favoriser la prise en compte de revendications politiques bien précises (l'indépendance du Khalistan, le devenir des ouvriers et/ou des riverains de l'usine AZF). Dans une telle optique stratégique, les réactions affectives manifestées telles le deuil, l'indignation, la nostalgie, etc. - parce qu'elles sont supposées obéir à des visées calculs d'ordre tactique - ne peuvent apparaitre dès lors que sous le jour d'émotions affectées, inauthentiques, peu sincères, voire même simulées. Les émotions manifestées seraient ici artificiellement apprêtées, arrangées et forcées, par des calculs qui leur préexisteraient et relèveraient d'une rationalité d'autant plus avancée qu'elle commanderait une expression des affects des plus contrôlées. Une figure interprétative alternative peut, en revanche, porter l'observateur à considérer que les émotions manifestées au cours des mobilisations de victimes - loin de se réduire à de simples moyens visant des buts qui les dépassent - doivent être envisagées comme ayant leur propre fin en soi. Les dispositifs mis en œuvre dans le cadre des mobilisations de victimes permettraient bel et bien d'éprouver et d'exprimer des émotions auxquelles les acteurs, en raison de leurs expériences sociales successives, attachent effectivement le plus grand prix. Prétendre rabattre des réactions affectives sur des finalités stratégiques relèverait d'ailleurs d'une forme d'imputation émique abusive sourde, non seulement aux exigences de la sociologie compréhensive, mais bien plus encore à la décence et à la pudeur la plus élémentaire ${ }^{6}$. Qui oserait douter, en effet, de la sincérité de la tristesse et de la colère des parents des victimes ? Ceux qui commémorent la disparition de leurs proches ne sont-ils pas réellement étreints par des

\footnotetext{
5 Sur la notion de dispositifs de sensibilisation, je me permets de renvoyer à Christophe Traïni (dir.), Émotions... mobilisation!, Paris, Presses de Science Po, à paraitre, février 2009.

${ }^{6}$ Selon Olivier de Sardan l'anthropologue tend à l'imputation émique abusive dès lors qu'il prétend rendre compte des comportements des individus étudiés en leur attribuant des motivations ou des logiques qui apparaissent, en fait, pour un observateur mieux informé ou plus attentif, en totale contradiction avec tout ce que l'on peut savoir sur ceux qui les mettent en œuvre. Jean-Pierre Olivier de Sardan, «La violence faite aux données. De quelques figures de la surinterprétation en anthropologie », Enquête, n³, 1996.
} 
Christophe TRAÏNI, «Les victimes entre émotions et stratégies », dans Sandrine Lefranc et Lilian Mathieu (dir.), Mobilisations de victimes, Presses Universitaires de Rennes, 2009.

sentiments les plus désintéressés qu'ils soient? Ne serait-il pas inconvenant de vouloir débusquer ici une quelconque instrumentalisation d'une douleur qui n'est en rien simulée ? Comme on peut le voir, l'inconvénient de ces deux formes d'interprétation alternatives est qu'elles nous invitent à rabattre les situations observées de manière quelque peu univoque, soit sur des usages stratégiques de registres victimaires, soit sur des manifestation incontrôlées d'émotion spontanés.

L'un des principaux intérêts des chapitres qui vont suivre réside dans le fait qu'ils nous exemptent de devoir opter ainsi pour une alternative au détriment de l'autre comme si rationalité et émotion devaient nécessairement et naturellement s'exclure. La finesse des descriptions empiriques qui étayent le propos des auteurs nous invite bien plutôt à relever dans quelle mesure les mobilisations de victimes se caractérisent par une inextricable imbrication d'élans d'apparence spontanée et de contrôles réflexifs des actions. Chacun des cas étudiés permet, en effet, d'entrevoir à quel point la manière dont les entrepreneurs de cause interpellent les publics ne peut que susciter des jeux d'émotion des plus complexes. L'expression "jeux d'émotions » doit être entendue au double sens du terme car il s'agit ici de désigner, non seulement la manifestation de séries, d'assortiments, d'ensembles composites de réactions affectives, mais encore des décalages, des défauts d'ajustement, des glissements subreptices, d'où résultent — en des termes moins métaphoriques — une pluralité de marges d'appréciation et d'action. La complexité de ces jeux d'émotions n'a rien pour surprendre puisqu'ils apparaissent, à l'analyse, extrêmement équivoques, ambivalents ${ }^{7}$ et précaires. Les émotions manifestées à travers les dispositifs propres aux causes victimaires étudiés se révèlent, en tout premier lieu, équivoques du fait de la multitude des publics éclectiques qu'ils parviennent à toucher. Ainsi, les commémorations de l'explosion de l'usine AZF étudiées par Stéphane Latté apparaissent éminemment équivoques dans la mesure où elles sont l'occasion d'éprouver des émotions hétérogènes qui doivent beaucoup à l'histoire et aux propriétés distinctes de chacun des groupes en présence: ouvriers de l'industrie chimique, propriétaires et riverains sinistrés à la suite de l'explosion, élus de la ville, professionnels des médias, ou bien encore psychologues dont le savoir-faire a été ici convoqué. Quant aux émotions suscitées par les évocations de l'assaut du Temple d'or analysées par Laurent Gayer, elles s'avèrent d'autant plus équivoques qu'elles participent à l'édification d'une mobilisation de nature transnationale. On imagine sans mal que les réactions affectives des populations sikhs vivant sur le même sol national que les armées indiennes qui ont massacré leurs proches ne se confondent nullement avec celles des sikhs de la diaspora qui, depuis Londres ou New York, ont été affectés par la nouvelle de l'évènement. Bien plus encore, la dénonciation d'un " génocide sikh » garantit d'autant moins la diffusion d'une émotion à la signification univoque et unanimement partagée que les dispositifs mis en place visent également à sensibiliser à la cause une opinion publique internationale bien peu au fait de l'histoire politique et de la culture sikh. L'équivocité des dispositifs victimaires semble même ici atteindre une telle intensité que l'on pourrait tout aussi bien parler d'un profond malentendu. Au regard disons faute de mieux de l'opinion publique internationale interpellée, l'évocation des victimes sikhs semble devoir susciter avant tout une combinaison de compassion et d'indignation, une demande de réparation, qui pointent rapidement en direction de ces modalités de règlement des conflits relativement pacifiées que constituent les procédures judiciaires et diplomatiques. Au regard de la culture et de «l'art du massacre » sikh, les victimes revêtent les traits des martyrs qui prescrivent, pour leur part, une identification à des modèles hérö̈ques, une émulation au combat, un désir de vengeance, bref une extension de la violence guerrière qui s'autorise à porter atteinte à l'intégrité physique des adversaires.

\footnotetext{
${ }^{7}$ Compte tenu de l'usage relâché qui peut parfois être fait de ces deux termes, il n'est sans doute pas inutile de rappeler leur signification exacte. On peut qualifier d'équivoque un objet ou une pratique dès lors qu'ils suscitent une pluralité de points de vue qui implique des évaluations bien distinctes. Un objet, une pratique, peuvent être qualifiés d'ambivalents dès lors qu'ils suscitent, à l'intérieur d'un seul et même point de vue, une combinaison de réactions affectives d'orientation divergente (amour/haine, confiance/crainte, admiration/mépris...).
} 
Christophe TRAÏNI, «Les victimes entre émotions et stratégies », dans Sandrine Lefranc et Lilian Mathieu (dir.), Mobilisations de victimes, Presses Universitaires de Rennes, 2009.

Pour ne rien perdre de la complexité des jeux d'émotions fomentés par les dispositifs des causes victimaires, il convient de remarquer comment ils peuvent également occasionner des attitudes ambivalentes au sein desquelles se combinent des réactions affectives d'orientation divergente. Le texte de Laurent Gayer suggère ainsi que la réaction des sikhs de la diaspora à la nouvelle de l'assaut du Temple d'or trahit des réactions affectives partiellement contradictoires. Les sentiments d'indignation qui résultent de la dénonciation du «génocide sikh » sembleraient $a$ priori manifester avant tout l'attachement à un futur Khalistan indépendant que les nationalistes appellent de leurs vœux. Pourtant, la mobilisation au nom des victimes outragées par l'inique répression orchestrée par l'armée indienne parait tout autant alimenter le souci d'affirmer publiquement dans le pays d'accueil l'appartenance à une minorité qui se doit d'être reconnue par les agences multiculturelles. Ainsi, la prescription du deuil et l'exigence de restauration de la dignité des sikhs relève moins d'un mythique projet de retour vers la terre des aïeuls que de la volonté de s'affirmer comme un sujet britannique ou américain à part entière. Se préoccuper du sort des victimes sikhs trahit autant l'attachement qu'une forme d'arrachement à l'égard du pays d'origine des parents. Dans le même ordre d'idée, il doit être évident à la lecture du texte de Stéphane Latté que les commémorations exclusives organisées par les ouvriers de l'usine AZF ne visent pas seulement à rendre hommage aux camarades de travail victimes de l'explosion. Une fonction moins manifeste semble bien destiner ces dispositifs commémoratifs à une forme de conjuration de la culpabilité que les riverains de l'usine attribuent aux professionnels d'une industrie chimique décrite comme irresponsable et meurtrière. De fait, le respect exigé à l'égard des victimes (majoritairement ouvrières) s'adresse autant aux morts qu'aux survivants qui doivent supporter le regard accusateur de leurs contemporains. A contrario, les commémorations mises en oeuvres par les riverains semblent bel et bien inciter autant à pleurer les victimes ouvrières qu'à condamner les activités dangereuses qu'elles pratiquaient de leur vivant.

$\mathrm{Du}$ fait de leur caractère ambivalent et équivoque, on ne s'étonnera pas que les jeux d'émotions sous-jacents aux mobilisations de victimes puissent également s'avérer instables, imprévisibles et partiellement incontrôlables. Ainsi, Laurent Gayer montre bien que l'évocation d'un " génocide sikh » n'a nullement interdit l'émergence d'une critique à travers laquelle certains sikhs, eux-mêmes, entendirent dénoncer, non seulement l'indifférence à l'égard de la domination exercée par les castes jats, mais plus encore l'inanité stratégique et le caractère diplomatiquement contre-productif du registre adopté. Alors même qu'ils étaient censés prescrire une solidarité et un recueillement des plus consensuels, les dispositifs mis en place ont pu éveiller des formes de ressentiment et d'hostilité. De même, il apparaît bien que les commémorations de l'explosion de l'usine AZF observées par Stéphane Latté ont été parfois marquées par des perturbations que les acteurs sociaux se sont aussitôt empressés de dénoncer en les qualifiant de "débordements", « consternants et indignes », qui constitueraient autant «d'insultes à la mémoire des victimes ». Certes, les dispositifs commémoratifs ont été soigneusement préparés afin d'assurer une coordination des émotions conforme à une définition de la situation qu'il aurait convenu de faire prévaloir: les lieux ont été soigneusement choisis, les entrées ont été filtrées, les prises de parole des parents de victimes ont été formatées, des psychologues ont été mandatés, etc... Pourtant, l'ensemble de ces précautions n'ont nullement suffit à interdire une évolution déroutante des commémoration au cours desquelles des émotions inopinées, bien différentes de celles qui étaient prescrites et attendues, finissent par se manifester.

Relever ainsi l'indétermination qui menace de troubler, à chaque instant, les émotions prescrites par les causes victimaires permet de mieux prendre la mesure du labeur incessant auquel leurs promoteurs sont astreints. Ces derniers, en effet, contrairement à ce que pourraient laisser penser certains paradigmes classiques de l'étude de l'action collective, ne se contentent jamais, ni de mobiliser des ressources, ni d'imposer un cadre d'interprétation, ni de tenter de bénéficier des opportunités offertes par des conjonctures strictement politiques. Bien plus fondamentalement, les entrepreneurs de cause se doivent de toucher leurs contemporains tout en 
Christophe TRAÏNI, « Les victimes entre émotions et stratégies », dans Sandrine Lefranc et Lilian Mathieu (dir.), Mobilisations de victimes, Presses Universitaires de Rennes, 2009.

évitant qu'une indétermination trop grande des émotions suscitées ne réduise à néant la possibilité de voir leurs revendications comprises et entendues. Certes, le ralliement du plus grand nombre s'accommode fort bien de la pluralité des interprétations et des manières d'apprécier les dispositifs de sensibilisation mis en œuvre. Cependant, la probabilité d'être soutenu par un large public s'amenuise grandement, en revanche, dès lors que les entrepreneurs de la cause s'avèrent incapables de juguler l'expression d'émotions sourdes, indicibles et socialement réprouvées, auxquelles ils peuvent, parfois bien malgré eux, frayer la voie: culpabilité des survivants, récriminations à l'égard des défunts, désir aveugle de vengeance, accusation de soutiens potentiels, plaisir de l'épanchement sans limite, etc... Les dispositifs mis en place se doivent d'éviter d'occasionner, auprès des publics apostrophés, embarras, gêne, perplexité, malaise, voire même une indignation qui pourrait évoluer vers une franche hostilité. De là découle la nécessité de ce travail de subordination des émotions sous des formes socialement légitimes que les chapitres suivants mettent remarquablement en exergue. Ainsi, les conflits entre les différents groupes sociaux concernés par l'explosion de l'usine AZF, s'ils leurs arrivent de se manifester lors de débordements sporadiques, sont pour le plus souvent tenus aux seuls euphémismes qu'autorisent les conventions sociales de l'hommage aux défunts, du recueillement, et des cérémonies funéraires. A contrario, les entrepreneurs de la cause sikh semblent avoir de grandes difficultés à rabattre l'ensemble des réactions affectives suscitées par le sort des assiégés du Temple d'or sur des conventions sociales reconnues aussi bien par les sikhs indiens, les membres de la diaspora, l'« opinion publique internationale » ou bien encore les agences onusiennes.

Signalons, enfin, que l'un des autres intérêts majeurs des propos présentés par nos auteurs est de démontrer la pertinence mais aussi les limites de la notion de choc moral proposée par James M. Jasper'. L'assaut du Temple d'or d'Amritsar en juin 1984, l'explosion de l'usine AZF, constituent bel et bien un choc moral à l'origine des mobilisations de victimes étudiées ici. Pourtant, il doit être évident que ces évènements — à moins d'apparaître comme des explications post hoc - ne peuvent être envisagés comme un facteur causal qui suffirait à rendre compte des formes d'activisme déployées. Certes, les chocs moraux en question impliquent bel et bien, comme le souligne le chercheur américain, une réaction très vive, viscérale, ressentie physiquement parfois même jusqu'à l'écoeurement, la nausée ou le vertige. Certes, ces évènements ont bien conduit les entrepreneurs des causes victimaires à jauger et juger la manière dont l'ordre présent du monde diverge scandaleusement avec les valeurs auxquelles ils adhèrent. Pourtant, les descriptions empiriques qui vont suivre attestent à quel point le travail qui consiste à transformer les réactions très vives, viscérales, éprouvées au cours du choc moral, en une cause collective fondée sur des valeurs collectives apparait — compte tenu justement de l'instabilité des jeux d'émotions — ni immédiat, ni gagné d'avance, ni irréversible. De là, découle la nécessité d'une analyse distinguant, d'une part les réactions affectives éprouvées antérieurement à l'activisme (notamment, mais non exclusivement, au cours du «choc moral»), et d'autre part les émotions qui se forment ou se renforcent à travers l'édification de la cause et qui, si elles constituent souvent une forme de réplique aux premières, produisent souvent des effets qui les dépassent très largement. Dans une telle optique, l'engagement en faveur des causes apparait comme l'élément d'un travail visant à peser sur les émotions qu'il est socialement bienvenu ou non d'éprouver et dont les tenants et aboutissants se doivent d'être interrogés à travers une échelle d'analyse à niveaux multiples : évolution des sentiments et des dispositions des militants, (re)définition d'opérateurs symboliques de regroupement («Nous»/«Eux»), mode de

\footnotetext{
${ }^{8}$ Sur la culpabilité des survivants, voir Primo Levi, Les Naufragés et les Rescapés : Quarante ans après Auschwitz, Gallimard, 1989.

9 Sur la notion de choc moral voir James M. Jasper, The Art of Moral Protest. Culture, Biography an Creativity in Social Movements, Chicago, University of Chicago Press, 1997 ; «The Emotions of Protest: Affective and Reactive Emotions In and Around Social Movements », Sociological forum, vol. 13, 1998. Je me permets également de renvoyer à mon article « choc moral » dans Olivier Fillieule, Lilian Mathieu, Cécile Péchu, Dictionnaire des mouvements sociaux, Presses de Sciences-po, à paraître.
} 
Christophe TRAÏNI, « Les victimes entre émotions et stratégies », dans Sandrine Lefranc et Lilian Mathieu (dir.), Mobilisations de victimes, Presses Universitaires de Rennes, 2009.

constitution et de résolution des conflits, altération des sensibilités collectives, production d'énoncés imposant un cadrage étayé sur des principes supérieurs communs, et enfin définition de griefs en direction des systèmes de la décision politique.

Christophe Traïni Institut d'Etudes Politiques d'Aix-en-Provence 